\title{
¿CuÁntas mesas debo tener? Sistemas de soporte de DeCISIONES PARA LA GESTIÓN DE RESTAURANTES
}

\section{Óscar Eduardo Sakay Rodríguez (Akio Sakai)}

\begin{abstract}
Resumen
El presente artículo muestra las ventajas que obtendría un restaurante al utilizar tecnologías de información (TI) no solo como herramienta operativa sino también como herramienta estratégica para la gestión. El restaurante donde se realizó el estudio cuenta con un modelo de gestión de operaciones, cuyos indicadores utilizan variables en términos de rentabilidad y utilización de recursos de manera independiente, es decir, no relacionadas; lo cual presenta desventajas ya que no es posible medir la rentabilidad en términos de la utilización de los recursos, como la de conocer la mezcla óptima de mesas. Responder estas inquietudes permitiría mejorar la toma de decisiones en el restaurante; por tal motivo se propuso utilizar un indicador mixto: REVPASH (revenue per available seat hour), el cual permite medir la rentabilidad del restaurante en términos de la utilización de sus recursos (mesas). Este indicador también se utilizó como punto de partida para solucionar el problema central de este artículo, para lo cual se construyó un Sistema de Soporte de Decisiones cuyo objetivo principal fue determinar la mezcla óptima de mesas, mejorando la rentabilidad del restaurante de acuerdo con la composición de los grupos de comensales, las horas de alta demanda del restaurante, entre otros aspectos.
\end{abstract}

Palabras clave: restaurante Revenue Management, REVPASH, sistemas de soporte de decisiones, gestión de mesas, Table Mix

\section{How many tables should i have? Decision support systems for restaurants management}

\section{Summary}

This article shows the benefits that a restaurant which uses Information Technologies (IT) as a strategic tool formanagement would get. The restaurant where the study was carried out, has a model for operations management, whose indicators use variables in terms of profitability and resource utilization in an independent manner, not related; the disadvantage is the inability to measure the profitability in terms of resource utilization. For this reason, a mixed indicator was used: REVPASH (revenue per available seat hour), which made it possible to measure the profitability of the restaurant in terms of the use of its resources. Taking this into consideration, a Decision Support System was developed that allowed us to determine the optimal combination of tables, improving the profitability of the restaurant according to the composition of the groups of guests, the high-demand times in the restaurant, among other things.

Key words: restaurant Revenue Management, REVPASH, decision support systems, management of tables, combination of tables 


\section{Introducción}

Hoy en día la industria gastronómica en el Perú ha sido transformada debido a los grandes festivales gastronómicos en todo el país, y su debido reconocimiento en el ámbito internacional. Esto ha generado que no solo el producto eleve su calidad y creatividad en la elaboración, sino también su presentación. Estos cambios que vive esta industria han traído nuevas oportunidades de negocios, los cuales deben ser aprovechados mediante la aplicación de las herramientas tecnológicas y tendencias administrativas que se han desarrollado en los últimos años a escala mundial.

En el mercado peruano, la oferta de herramientas de información y tecnología que brindan valor a esta industria se encuentra limitada a un solo tipo, que es de carácter operacional, las cuales se denominan Punto de Venta (POS, Point of Sale); su función principal es agilizar los procesos productivos al disminuir los tiempos en las operaciones, por ejemplo el tiempo que transcurre desde que se realiza un pedido hasta que este entra en cocina, el tiempo en el que se expide el cierre de cuenta hasta la emisión de la boleta de venta, etcétera. Sin embargo, debido a las limitantes de diseño, la información detallada que contiene un recibo de consumo no es explotada en su totalidad, dejando de lado grandes oportunidades para generar mayor rentabilidad y valor al negocio.

Lamentablemente muchos restaurantes limeños son ajenos a las nuevas tendencias administrativas, por lo que la inversión en tecnología de información se encuentra estancada en el contexto mencionado. Por otro lado, la oferta de sistemas de información que incorporen estas nuevas técnicas en el mercado local es prácticamente nula, motivo por el cual la industria no ha podido evolucionar en el plano administrativo.

\section{Objetivos del estudio}

A continuación se presentan el objetivo principal y los objetivos específicos planteados para el presente estudio:

\subsection{Objetivo principal}

El objetivo principal consiste en diseñar y elaborar una herramienta y modelo de gestión para la mejora en la composición de la mezcla de mesas en un restaurante.

\subsection{Objetivos específicos}

E1: Determinar los horarios de alta y baja demanda del restaurante. 
E2: Estimar los tiempos de estadía de los comensales.

E3: Generar estrategias de gestión del cliente en función a los horarios de demanda y el tiempo de estadía de los comensales.

E4: Determinar el impacto en los ingresos del restaurante según las diferentes mezclas de mesas al implementar políticas de gestión del cliente.

\section{Marco teórico}

\subsection{Revenue Management en restaurantes}

Cada vez que una persona desea vender algún artículo, debe encarar interrogantes como ¿qué día realizar la venta?, ¿qué precio solicitar por el artículo?, y en algunos casos ¿cuándo subir o bajar los precios?, enfrentándose a varias decisiones que tendrán un impacto directo sobre la transacción. Cualquier persona que haya enfrentado este tipo de decisiones, sin importar lo que se está ofreciendo, conoce la incertidumbre que existe de por medio (Talluri y van Ryzin, 2004).

En el caso de las grandes empresas, estas buscan vender cuando las condiciones del mercado o condiciones externas son las más favorables, prevaleciendo aún interrogantes de carácter interno, como la determinación del precio del producto o servicio, así como la predicción de la demanda. Debido a la incertidumbre de la demanda, la fijación de los precios no debe ser tan alta para no desalentar a los potenciales compradores, ni tan bajo como para que el margen de ganancia se vea reducido de manera drástica. Además, las grandes empresas enfrentan decisiones más complejas a las ya mencionadas, debido a que la mayoría tiene a sus clientes en diferentes segmentos y canales. Es entonces cuando las interrogantes sobre el diseño del producto o servicio surgen, con el fin de evitar el "canibalismo" entre segmentos y canales.

El Revenue Management (RM) se encarga de estudiar y asistir a este tipo de decisiones de gestión de la demanda o Demand-Management Decisions (DMD), enlazando los procesos de gestión empresarial con su interface de mercado respectiva, teniendo como objetivo el aumento de la rentabilidad del negocio. El RM puede ser visto como un complemento de la gestión de cadena de suministro, donde usualmente el objetivo es minimizar los costos de producción y de entrega al cliente.

Dependiendo de la industria, la práctica del RM recibe otras denominaciones tales como Yield Management (industria aeronáutica), Pricing and Revenue Management, Pricing and Revenue Optimization, Revenue Process Optimization, Demand Management, etcétera (Talluri y van Ryzin, 2004). Sin embargo, se utilizará el término estándar Revenue Management para referirse a este amplio rango de 
técnicas, decisiones, métodos, procesos y tecnologías que interactúan con la gestión de la demanda.

En la industria de los restaurantes es posible la aplicación del RM debido a que el tipo de decisiones que se deben afrontar pertenecen al grupo de las DMD. Lamentablemente, la aplicación del RM se realiza en un plano táctico, por lo que tiene poco impacto en las estrategias de este tipo de negocio. Sin embargo, conocer las particularidades de esta industria y los factores que la diferencian de otras es pieza clave para que la aplicación del RM pase de un plano táctico a uno estratégico. Entre las características que tiene el RM en restaurantes, de acuerdo con Kimes (2004), destacan las siguientes:

a) Capacidad relativamente fija.- La capacidad de un restaurante suele ser medida por el número de sillas, el tamaño de la cocina, los ítems del menú o los niveles de personal existente. Muchos tomadores de decisiones suelen utilizar y optimizar la capacidad al tratar de asignar todos los asientos disponibles y rotar las mesas con la mayor rapidez. Sin embargo, este esfuerzo puede ser opacado por problemas en la cocina, como falta de personal de servicio. Otro factor importante por el que la capacidad es relativamente fija se debe al tamaño de las mesas, ya que estas, al tener menor capacidad (mesas para dos personas), tienden a quitar espacio en el establecimiento, esto hace que sea importante contar con la mezcla apropiada de mesas para definir la capacidad.

b) Manejo de la demanda como inventario.- La demanda en un restaurante puede ser vista como inventario, mediante la toma de reservas o la generación de colas de clientes. Muchos restaurantes toman reservaciones o ventas por adelantado para generar demanda y con ello inventario. Otros restaurantes operan bajo reservas con el fin de asegurar su inventario y sus ventas. Las reservas suelen ser muy valiosas ya que con ellas los tomadores de decisiones tienen la oportunidad de vender y controlar el inventario con anticipación, aceptando o rechazando reservas, dependiendo de la demanda del restaurante.

c) Demanda variable en el tiempo.- La demanda de un restaurante está prácticamente constituida por dos tipos de clientes: los que mediante reservaciones ingresan al restaurante, y los clientes walk in o que simplemente ingresan de manera aleatoria. Estas dos formas de demanda pueden ser gestionadas con diferentes estrategias, teniendo en cuenta su dependencia según la hora, el día de la semana y el mes del año.

d) Clientes segmentables.- Así como en otras industrias, los restaurantes también pueden segmentar a sus clientes debido a su sensibilidad respecto al precio. Por ejemplo: una familia con niños podrían estar dispuestos a 
cambiar tiempo de consumo o ubicación dentro del restaurante por un descuento. Por otro lado, otros clientes cuya sensibilidad al precio sea casi nula, podrían estar dispuestos a pagar un cargo extra por tener la mesa de su elección.

e) Perecibilidad del producto.- Cuando hablamos de productos perecederos en un restaurante, se suele pensar en los insumos de los ítems de la carta, sin embargo, de acuerdo con el RM el producto que se oferta en un restaurante no son los ítems sino el tiempo en el cual un asiento está siendo utilizado por un cliente, el cual se mide monetariamente mediante el consumo del cliente.

En la actualidad, la forma más común de medir y evaluar la rentabilidad en los restaurantes es mediante el ticket promedio, el número de rotaciones de mesas por unidad de tiempo y los porcentajes de costos que el restaurante puede minimizar. Lamentablemente, estas mediciones no capturan suficiente información sobre el desempeño de la rentabilidad del restaurante. Por ejemplo, el ticket promedio no toma en cuenta la información necesaria para medir la rentabilidad en términos de capacidad. Por tal motivo, el RM propone al REVPASH como indicador mixto para realizar lecturas más completas.

El REVPASH se encarga de medir el ratio en el cual se genera la rentabilidad y captura los cambios monetarios entre los tickets de consumo y el uso de instalaciones del restaurante. Si la ocupación de asientos aumenta, aunque el ticket promedio disminuya, el REVPASH puede que no varíe. De similar forma, si el ticket promedio aumenta y la ocupación de asientos disminuye, el REVPASH puede mantenerse.

\subsection{Cálculo del REVPASH}

La forma más práctica de calcular el REVPASH es dividiendo los ingresos, es decir, la sumatoria de los consumos, del período en análisis entre el número de asientos disponibles, en cada unidad de tiempo, como se puede apreciar en la siguiente fórmula:

$$
\mathrm{REVPASH}=\frac{T}{S^{*} A}
$$

Donde:

T: Sumatoria de todos los tickets del período por calcular,

S: Número de asientos que tiene el restaurante,

A: Unidad de tiempo. 
Por ejemplo: un restaurante, el cual dispone de 100 asientos, presenta una sumatoria de tickets de S/.3000 entre las 12:00 horas y las 14:00 horas. Entonces, su REVPASH por el período de 2 horas es igual a:

\section{REVPASH $=\frac{\frac{S / .3000}{100 \text { asientos }}}{2 \text { horas }}=\mathrm{S} / .15$ por asiento durante cada una de las 2 horas}

En conclusión, para tener éxito en la práctica del RM es necesario desarrollar un programa de RM en el cual el tomador de decisiones entienda las condiciones y el desempeño del restaurante, y le permita evaluar los factores que impactan directa o indirectamente dicho desempeño, y al entender estos factores determinar cómo mejorar el REVPASH generando diferentes estrategias. Una vez que estas estrategias han sido implementadas, se deben monitorear los efectos del cambio, realizando los ajustes que sean necesarios.

\subsection{Gestión de mesas (Tab/e Mix)}

La utilización de asientos en la industria de los restaurantes puede ser fijada de tres formas básicas: aumentando la demanda, disminuyendo la duración de los ciclos o brindando una mejor mezcla de mesas. Asimismo, se puede usar una combinación de las formas señaladas.

Como herramienta para gestionar la utilización de los asientos, muchos restaurantes de todo el mundo suelen utilizar las reservas de mesas. Sin embargo, en la coyuntura social que tiene el Perú esta táctica es poco utilizada. Los restaurantes que operan bajo reservaciones pueden programar y ubicar al cliente en una mesa adecuada con la cantidad de integrantes del grupo para el que se ha realizado la reserva, Person at table (PAX de clientes), mejorando la utilización de los recursos. Por otro lado, para aquellos restaurantes que no operan bajo esta modalidad, la clave para maximizar la utilización de asientos recae en encontrar la mejor mezcla de mesas dependiendo del día y la hora, es decir, si un restaurante asegura que la mezcla de mesas es igual o muy aproximada al tamaño del grupo o PAX, puede obtener un mayor porcentaje de utilización de los asientos (Hwang y Yoon, 2009).

Se define a la mezcla de mesas como el total de mesas con " $n$ " asientos que se pueden ubicar dentro del restaurante, es decir el número de mesas asignadas para dos, cuatro, seis personas, etcétera. Se supone que cualquier variación que se ejecute en la mezcla de mesas puede tener un impacto en el REVPASH. Para ello, el uso de herramientas de gestión de mesas puede facilitar la información necesaria para determinar la mezcla óptima dependiendo del día y la hora, entre otros factores. 
La gestión de mesas en un restaurante también se encarga de planificar el área donde el servicio será realizado. Comprende no solo la distribución de las mesas sino también su capacidad, la gestión de colas, el monitoreo de las mesas, etc. Actualmente existen numerosas herramientas de información que asisten de manera gráfica a la gestión misma; estas herramientas también pueden ser utilizadas para medir y costear las labores del servicio, medir la duración de los ciclos y controlar las operaciones. Soluciones como las que ofrece la compañía MICROS System brindan cierta información referente a la mezcla de las mesas. Al ser conectadas al POS del restaurante estas herramientas podrían capturar información referente a la hora de entrada y salida de los clientes, el PAX, entre otros. Si se procesa esta información junto con la información histórica del restaurante, mediante algoritmos de optimización o simulación, daría como resultado la mezcla óptima, maximizando la utilización de los asientos y mejorando la rentabilidad del negocio (Kimes y Thompson, 2004).

El problema que conlleva el uso de este tipo de herramientas se encuentra en que es una caja negra, es decir, opera bajo algoritmos de optimización que no son accesibles, y puede ser el caso de que dicho algoritmo no modele adecuadamente la realidad del restaurante; por lo cual, la creación de diferentes escenarios se vuelve dificultosa. Por otro lado, modelar la realidad de un restaurante mediante una simulación brinda como ventaja la creación de numerosos escenarios, que permiten analizar el rendimiento de cada uno de ellos, y mediante la experimentación dentro del modelo mismo, posibilita innovar en los diferentes fenómenos que ocurren, sin la necesidad de realizar una implementación directa para pronosticar resultados.

3.

\section{Caso de análisis}

El estudio fue aplicado en un restaurante situado en el distrito de Miraflores, en la ciudad de Lima, Perú. El restaurante inició sus operaciones en el mes de diciembre de 2011, atendiendo a sus clientes bajo el siguiente formato: durante el día como restaurante y durante la noche como un pub-bar. Por decisión de los socios fundadores, dentro de su carta se ofrecen platos de comida criolla, marina y fusión (japonesa contemporánea). Debido a que el restaurante se encuentra ubicado en una calle secundaria, su público objetivo durante la semana consiste en las personas que trabajan por los alrededores del local. Durante los fines de semana, principalmente se atiende a familias y parejas, en vez de grupos turísticos como era de esperarse debido a su ubicación. El horario de atención al público es de lunes a sábado, desde las 11:00 horas hasta las 14:00 horas, con cierre temporal para cambio de ambientación desde las 17:00 horas hasta las 19:00 horas. 
Como punto de partida para el análisis se realizó el cálculo del REVPASH, indicador que se tomará como referencia para medir la rentabilidad del restaurante; el mencionado indicador se obtuvo restando la hora de pago y salida del cliente con la respectiva hora de toma del primer pedido, el cual fue anotado en las comandas de los meseros; asimismo, se recabó el PAX del grupo, el número de mesa y cada uno de los ítems solicitados. El período de recopilación de los datos fue de 25 semanas y los datos recabados fueron procesados utilizando una herramienta informática, mapeando el REVPASH toda la semana; los resultados obtenidos se presentan en la tabla 1:

Tabla 1. REVPASH del ejemplo de aplicación

\begin{tabular}{|c|c|c|c|c|c|c|c|}
\hline Hora & Lunes & Martes & Miércoles & Jueves & Viernes & Sábado & Domingo \\
\hline 12:00 - 13:00 & $\mathrm{S} / 7.36$ & $\mathrm{~S} / .6 .58$ & $\mathrm{~S} / .7 .91$ & $\mathrm{~S} / 7.21$ & $\mathrm{~S} / .5 .05$ & $\mathrm{~S} / .2 .27$ & $\mathrm{~S} / .0 .00$ \\
\hline $13: 00-14: 00$ & $\mathrm{~S} / .72 .86$ & $\mathrm{~S} / .57 .62$ & $\mathrm{~S} / .67 .77$ & $\mathrm{~S} / .82 .45$ & $\mathrm{~S} / .82 .12$ & $\mathrm{~S} / .29 .58$ & $\mathrm{~S} / .4 .19$ \\
\hline 14:00 - 15:00 & $\mathrm{S} / .80 .81$ & $\mathrm{~S} / 75.12$ & $\mathrm{~S} / 76.19$ & $\mathrm{~S} / .82 .37$ & $\mathrm{~S} / .97 .25$ & $\mathrm{~S} / .54 .05$ & $\mathrm{~S} / .4 .35$ \\
\hline $15: 00-16: 00$ & $\mathrm{~S} / .35 .63$ & $\mathrm{~S} / 24.06$ & $\mathrm{~S} / .18 .74$ & $\mathrm{~S} / .25 .44$ & $\mathrm{~S} / 28.79$ & $\mathrm{~S} / .51 .44$ & $\mathrm{~S} / .3 .08$ \\
\hline $16: 00-17: 00$ & $\mathrm{~S} / 5.03$ & $\mathrm{~S} / .9 .00$ & $\mathrm{~S} / .1 .87$ & $\mathrm{~S} / 2.61$ & $\mathrm{~S} / .7 .29$ & $\mathrm{~S} / .18 .76$ & $\mathrm{~S} / .0 .62$ \\
\hline 17:00 - 18:00 & $\mathrm{S} / 1.71$ & $\mathrm{~S} / .2 .31$ & $\mathrm{~S} / .0 .00$ & $\mathrm{~S} / 1.14$ & $\mathrm{~S} / .0 .29$ & $\mathrm{~S} / .2 .51$ & $\mathrm{~S} / .4 .46$ \\
\hline $18: 00-19: 00$ & $\mathrm{~S} / .0 .00$ & $\mathrm{~S} / .0 .00$ & $\mathrm{~S} / .0 .00$ & $\mathrm{~S} / 0.00$ & $\mathrm{~S} / .0 .00$ & $\mathrm{~S} / 0.42$ & $\mathrm{~S} / .0 .00$ \\
\hline
\end{tabular}

Elaboración propia.

En la tabla se aprecia que el valor del REVPASH en los días domingos es muy bajo, lo que se traduce como un pobre desempeño de los asientos en comparación con los días anteriores. Esto se debe a que el restaurante se encuentra ubicado en una zona frecuentada en su mayoría por oficinistas, por lo que su flujo de clientes dominicales es muy bajo. Por otro lado, se observa que de lunes a viernes el indicador supera los S/.50 por asiento entre las 13:00 y las 15:00 horas; similar situación se presenta, pero en menor medida, los días sábados, de 14:00 a 16:00 horas. Otro dato importante que brinda la tabla son las horas de alta demanda del restaurante, que en este caso son un total de 12 horas semanales, lo que se encuentra relacionado con las dos horas de alta demanda durante seis días de la semana.

Al tener precios fijos en los ítems, el REVPASH solo puede incrementarse al reducir la duración de cada ciclo de atención al cliente, aumentando la cuenta promedio o la utilización de asientos. Después de filtrar los datos recabados se determinó que el ticket promedio fue de S/.54.43, y la duración promedio de cada ciclo fue de 63.72 minutos, independientemente del PAX durante las 25 semanas del estudio. Con esta información se puede afirmar de manera hipotética que el 
ingreso máximo que el restaurante puede generar es de S/.32 596.50, el cual fue calculado de la siguiente manera:

$$
\text { Ingresos }=\left(\frac{H P * M}{M P}\right) * A * C P * U
$$

Donde:

HP: Número de horas pico por semana (12 horas),

M: Cantidad de minutos que tiene nuestra unidad de medición (60 minutos),

MP: Promedio, en minutos, que tarda en completarse un ciclo de atención (63.72 minutos),

A: Número de asientos que tiene el restaurante (53 asientos),

CP:Ticket promedio del restaurante (S/. 54.43),

U: Utilización de los asientos; que para este caso se consideró el $100 \%(U=1)$.

En caso de que el ciclo de atención se redujera en cinco minutos, es decir, a un tiempo promedio de 57.72 minutos, los nuevos ingresos serían de S/.35 372.08, lo que equivale a un incremento de S/.2775.58 en los ingresos. Con este ejemplo se confirma que reduciendo la duración del ciclo de atención tiene un impacto directo en la rentabilidad; sin embargo, se mencionó que el aumento en la utilización también tiene un impacto. Por tal motivo se calculó la utilización que tuvieron las mesas del restaurante durante el período de estudio, lo cual se puede apreciar en las tablas 2 y 3 :

Tabla 2. Porcentaje de utilización de mesas

\begin{tabular}{|c|c|c|c|c|c|c|c|}
\hline Hora & $\begin{array}{c}\text { Lunes } \\
\%\end{array}$ & $\begin{array}{c}\text { Martes } \\
\%\end{array}$ & $\begin{array}{c}\text { Miércoles } \\
\%\end{array}$ & $\begin{array}{c}\text { Jueves } \\
\%\end{array}$ & $\begin{array}{c}\text { Viernes } \\
\%\end{array}$ & $\begin{array}{c}\text { Sábado } \\
\%\end{array}$ & $\begin{array}{c}\text { Domingo } \\
\%\end{array}$ \\
\hline 12:00 - 13:00 & 11 & 11 & 11 & 11 & 11 & 0 & 0 \\
\hline 13:00 - 14:00 & 56 & 44 & 44 & 44 & 78 & 11 & 11 \\
\hline $14: 00-15: 00$ & 89 & 78 & 78 & 89 & 100 & 2 & 11 \\
\hline $15: 00-16: 00$ & 44 & 22 & 33 & 33 & 22 & 44 & 11 \\
\hline $16: 00-17: 00$ & 11 & 11 & 11 & 22 & 22 & 33 & 11 \\
\hline $17: 00-18: 00$ & 11 & 11 & 0 & 11 & 11 & 11 & 11 \\
\hline 18:00 - 19:00 & 0 & 0 & 0 & 0 & 11 & 11 & 0 \\
\hline
\end{tabular}

Elaboración propia. 
Tabla 3. Porcentaje de utilización de la barra

\begin{tabular}{|c|c|c|c|c|c|c|c|}
\hline Hora & $\begin{array}{c}\text { Lunes } \\
\%\end{array}$ & $\begin{array}{c}\text { Martes } \\
\%\end{array}$ & $\begin{array}{c}\text { Miércoles } \\
\%\end{array}$ & $\begin{array}{c}\text { Jueves } \\
\%\end{array}$ & $\begin{array}{c}\text { Viernes } \\
\%\end{array}$ & $\begin{array}{c}\text { Sábado } \\
\%\end{array}$ & $\begin{array}{c}\text { Domingo } \\
\%\end{array}$ \\
\hline 12:00 - 13:00 & 0 & 7 & 0 & 0 & 7 & 7 & 0 \\
\hline 13:00 - 14:00 & 0 & 7 & 7 & 13 & 27 & 7 & 0 \\
\hline $14: 00-15: 00$ & 0 & 0 & 7 & 13 & 33 & 13 & 0 \\
\hline $15: 00-16: 00$ & 0 & 0 & 0 & 7 & 13 & 0 & 0 \\
\hline $16: 00-17: 00$ & 0 & 0 & 0 & 0 & 0 & 0 & 0 \\
\hline $17: 00-18: 00$ & 0 & 0 & 0 & 0 & 0 & 0 & 0 \\
\hline 18:00 - 19:00 & 0 & 0 & 0 & 0 & 0 & 0 & 0 \\
\hline
\end{tabular}

Elaboración propia.

Como se puede apreciar, de lunes a viernes la utilización de mesas supera el $50 \%$ entre las 14:00 y las 15:00 horas, mientras que el resto del tiempo no supera el 50 \%, con excepción del día lunes, de 13:00 a 14:00 horas. La utilización promedio de las mesas durante el horario de atención como restaurante durante las 25 semanas fue del $55.42 \%$.

Al aplicar la fórmula descrita se observa que los ingresos generados por las mesas, con la utilización promedio de $55.42 \%$, es de S/.18 065, mientras que si se eleva la utilización en 15 \% el nuevo ingreso sería de S/.22 954, es decir un aumento de S/.4889. Aparte de estos ejemplos, se calcularon diferentes variantes de aumento de utilización y disminución de la duración del ciclo, dando como resultado casi siempre que aumentar la utilización tiene un mayor impacto en la rentabilidad que disminuir la duración del ciclo. Para ello se elaboró una tabla donde se cruzaron porcentajes de utilización con diferentes duraciones de ciclo (véase la tabla 4), ya que ello permitirá otorgarle al administrador una pauta para generar pronósticos de ventas de acuerdo al día de la semana y con ello elevar el REVPASH (Kimes et al., 2002).

\begin{tabular}{|c|c|c|}
\hline $\begin{array}{c}\text { Capacidad } \\
\text { (asientos) }\end{array}$ & $\begin{array}{c}\text { Horas pico } \\
\text { semanales }\end{array}$ & $\begin{array}{c}\text { Ticket } \\
\text { promedio }\end{array}$ \\
\hline 53 & 12 & 54.43 \\
\hline
\end{tabular}


Tabla 4. Ingresos potenciales mediante cambios en la utilización y la duración del ciclo de atención

\begin{tabular}{|c|c|c|c|c|c|c|c|}
\hline & \multicolumn{7}{|c|}{ Ingresos potenciales por semana (S/.) } \\
\cline { 2 - 9 } $\begin{array}{c}\text { Duración ciclo } \\
\text { promedio }\end{array}$ & $100 \%$ & $90 \%$ & $80 \%$ & $70 \%$ & $60 \%$ & $50 \%$ & $40 \%$ \\
\hline 63 & 32969.03 & 29672.13 & 26375.22 & 23078.32 & 19781.42 & 16484.51 & 13187.61 \\
\hline 62 & 33500.79 & 30150.71 & 26800.63 & 23450.55 & 20100.47 & 16750.39 & 13400.31 \\
\hline 61 & 34049.98 & 30644.98 & 27239.98 & 23834.99 & 20429.99 & 17024.99 & 13619.99 \\
\hline 60 & 34617.48 & 31155.73 & 27693.98 & 24232.24 & 20770.49 & 17308.74 & 13846.99 \\
\hline 59 & 35204.22 & 31683.80 & 28163.37 & 24642.95 & 21122.53 & 17602.11 & 14081.69 \\
\hline 58 & 35811.19 & 32230.07 & 28648.95 & 25067.83 & 21486.71 & 17905.59 & 14324.47 \\
\hline 57 & 36439.45 & 32795.51 & 29151.56 & 25507.62 & 21863.67 & 18219.73 & 14575.78 \\
\hline 56 & 37090.16 & 33381.14 & 29672.13 & 25963.11 & 22254.09 & 18545.08 & 14836.06 \\
\hline 55 & 37764.52 & 33988.07 & 30211.62 & 26435.17 & 22658.71 & 18882.26 & 15105.81 \\
\hline 54 & 38463.87 & 34617.48 & 30771.09 & 26924.71 & 23078.32 & 19231.93 & 15385.55 \\
\hline 53 & 39189.60 & 35270.64 & 31351.68 & 27432.72 & 23513.76 & 19594.80 & 15675.84 \\
\hline 52 & 39943.25 & 35948.92 & 31954.60 & 27960.27 & 23965.95 & 19971.62 & 15977.30 \\
\hline 51 & 40726.45 & 36653.80 & 32581.16 & 28508.51 & 24435.87 & 20363.22 & 16290.58 \\
\hline 50 & 41540.98 & 37386.88 & 33232.78 & 29078.68 & 24924.59 & 20770.49 & 16616.39 \\
\hline
\end{tabular}

Elaboración propia.

\section{Propuesta de solución}

Hasta el momento se ha determinado que para incrementar el REVPASH se puede lograr mediante el aumento de la utilización de mesas. Sin embargo, no siempre la capacidad de cada mesa se ajusta a la composición de los grupos de comensales, para poder determinar la mezcla de mesas que optimice la utilización, y como propuesta de solución se optó por el desarrollo de un modelo de simulación, tal como lo recomiendan Padilla y Cossa (2011).

\subsection{Consideraciones para el modelo de simulación}

\subsubsection{Distribución del restaurante}

El primer paso para la construcción del modelo de simulación fue conocer la mezcla actual de las mesas y sillas del restaurante, la cual denominaremos "As Is" y consta de: una mesa para dos personas, seis mesas para cuatro personas, dos mesas para seis 
personas y una barra con quince asientos personales. La mencionada distribución se representa en la figura 1.

Figura 1. Plano del restaurante

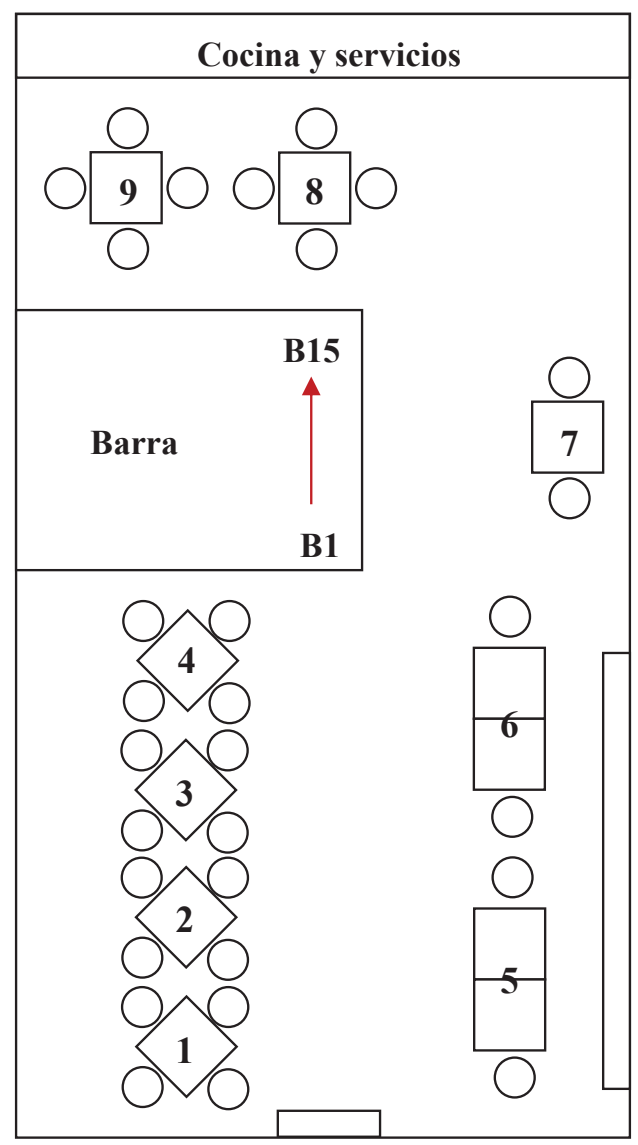

Elaboración propia.

\subsubsection{Intervalos de arribo de los clientes}

El intervalo de arribo de los clientes constituye los datos de entrada en el modelo de simulación, para lo cual mediante observación y medición directa de los tiempos, se determinan los tiempos entre los arribos de cada cliente durante los diferentes días de la semana. En base a una muestra de 300 arribos, los cuales fueron procesados mediante el software ARENA, y se obtuvieron las distribuciones estadísticas respectivas, las cuales presentan en la tabla 5. 
Tabla 5. Distribución de los tiempos entre arribos de clientes

\begin{tabular}{|c|c|}
\hline Día de la semana & Distribución estadística \\
\hline Lunes & Exponencial (16.2) \\
\hline Martes & Exponencial (13.0) \\
\hline Miércoles & Exponencial (15.4) \\
\hline Jueves & Exponencial (17.3) \\
\hline Viernes & Exponencial (14.5) \\
\hline Sábado & Exponencial (19.4) \\
\hline
\end{tabular}

Elaboración propia.

No se consideró el día domingo en la simulación, ya que los dueños decidieron no atender en el día mencionado, debido a la poca asistencia de clientes.

\subsubsection{Composición de los grupos (PAX)}

La composición de los grupos de clientes que frecuentan el restaurante (véase la tabla 6) se obtuvo a través de las comandas emitidas por los mozos. La mayoría de grupos de clientes, el $72 \%$ del total, corresponden a grupos de clientes constituidos por una o dos personas. El $14 \%$ corresponde a grupos de tres personas, mientras que el $7 \%$, 3 \% y 4 \% restante corresponde a grupos de cuatro, cinco y seis o más personas, respectivamente.

Tabla 6. Composición del PAX

\begin{tabular}{|c|c|}
\hline PAX & Porcentaje \\
\hline 1 PAX & 28 \\
\hline 2 PAX & 44 \\
\hline 3 PAX & 14 \\
\hline 4 PAX & 7 \\
\hline 5 PAX & 3 \\
\hline $6++$ PAX & 4 \\
\hline
\end{tabular}

Elaboración propia.

La composición del PAX que frecuenta el restaurante fue una de las señales que indicó que se debería mejorar la gestión de mesas, ya que seis de las nueve mesas están diseñadas para atender grupos de cuatro personas, sin embargo la mayor cantidad de clientes vienen en grupos de dos personas. Mientras parece obvio que 
el restaurante debería incrementar su número de mesas para atender grupos más pequeños, la interrogante frente a la mezcla óptima no puede ser respondida en su totalidad, ya que existen variaciones entre los arribos y diferentes duraciones en el ciclo de atención, tal como se aprecia en la tabla 7.

Tabla 7. Consumo y tiempo promedio por PAX

\begin{tabular}{|c|c|c|}
\hline PAX & Consumo promedio & $\begin{array}{c}\text { Tiempo promedio } \\
\text { (min.) }\end{array}$ \\
\hline 1 PAX & $\mathrm{S} / .22 .53$ & 47.34 \\
\hline 2 PAX & $\mathrm{S} / 47.44$ & 53.60 \\
\hline 3 PAX & $\mathrm{S} / .70 .02$ & 60.11 \\
\hline 4 PAX & $\mathrm{S} / .92 .12$ & 64.29 \\
\hline 5 PAX & $\mathrm{S} / .113 .00$ & 66.93 \\
\hline $6++$ PAX & $\mathrm{S} / .142 .12$ & 70.88 \\
\hline
\end{tabular}

Elaboración propia.

Se identificó que un factor limitante fue el espacio físico que dispone el restaurante, motivo por lo que cualquier tipo de mezcla debe contemplar el mismo número de asientos para que los ingresos se vean reflejados en el incremento del REVPASH.

\subsubsection{Tiempo de las operaciones}

La información referente a los diferentes tiempos que comprende cada proceso fue captada utilizando un reporte del POS, y cruzándola con mediciones directas en dichos procesos. Se determinó que los tiempos de atención por parte de los mozos sigue una distribución triangular, con parámetros $(0,3.91,7)$ minutos; los tiempos de preparación en cocina, consumo, pago y retiro de los clientes presentan también una distribución triangular (tablas 8, 9 y 10), y por último, el tiempo de limpieza de las mesas responde a una distribución triangular con parámetros $(0.31,1.89,3.61)$ minutos.

Tabla 8. Distribución de los tiempos de preparación en cocina

\begin{tabular}{|c|c|}
\hline PAX & Distribución triangular (Min, Moda, Max) \\
\hline 1 PAX & Triangular $(0.5,10.1,14)$ \\
\hline 2 PAX & Triangular $(8,11.8,16)$ \\
\hline 4 PAX & Triangular $(11,15.8,20)$ \\
\hline $6++$ PAX & Triangular $(8,17.5,25)$ \\
\hline
\end{tabular}

Elaboración propia. 
Tabla 9. Distribución de los tiempos de consumo por PAX

\begin{tabular}{|c|c|}
\hline PAX & Distribución triangular (Min, Moda, Max) \\
\hline 1 PAX & Triangular $(24.5,35,42.5)$ \\
\hline 2 PAX & Triangular $(26.5,42,60.5)$ \\
\hline 4 PAX & Triangular $(26.5,43,81.5)$ \\
\hline $6++$ PAX & Triangular $(44.5,63,85.5)$ \\
\hline
\end{tabular}

Elaboración propia.

Tabla 10. Distribución de los tiempos de pago y salida del PAX

\begin{tabular}{|c|c|}
\hline PAX & Distribución triangular (Min, Moda, Max) \\
\hline 1 PAX & Triangular $(0.45,1.01,1.56)$ \\
\hline 2 PAX & Triangular $(1,2.22,3)$ \\
\hline 4 PAX & Triangular $(3,4.88,8)$ \\
\hline $6++$ PAX & Triangular $(3,6.07,9)$ \\
\hline
\end{tabular}

Elaboración propia.

Las distribuciones se calcularon tomando en cuenta que cada PAX tiene un comportamiento diferente, por lo tanto, en el modelo de simulación se modelaron por separado. Los tiempos de atención de los mozos se modelaron sin tomar en cuenta la composición del PAX al que atienden, ya que la variación fue demasiado corta como para tomarla en consideración, de similar forma se calculó el tiempo de limpieza de las mesas; por otro lado, para calcular las distribuciones de la cocina se tomaron diferentes muestras de acuerdo al orden de los pedidos.

\subsubsection{Reglas de decisión}

Dada la mezcla de clientes que frecuentan el restaurante, la mezcla de mesas actual se encuentra muy desbalanceada. Se sabe, además, que el restaurante no aplica ninguna política de gestión del cliente; por lo que se puede asumir lo siguiente en relación a la forma de ubicar a los grupos de clientes (véanse las tablas 11 y 12), que llegan al restaurante:

i. Un grupo de una o dos personas puede sentarse en la barra, y en las mesas para dos, cuatro y seis personas.

ii. Un grupo de tres o cuatro personas puede sentarse en la barra, y en las mesas para cuatro y seis personas.

iii. Un grupo de cinco o seis personas puede sentarse en la barra o en las mesas para seis personas. 
Tabla 11. Combinaciones de PAX vs tipo de mesa

\begin{tabular}{|c|c|}
\hline PAX & Tipo de mesa \\
\hline 1 & Barra, $2,4,6$ \\
\hline 2 & $2,4,6$ \\
\hline 3 & 4,6 \\
\hline 4 & 4,6 \\
\hline 5 & 6 \\
\hline 6 & 6 \\
\hline
\end{tabular}

Elaboración propia.

Tabla 12. Porcentaje de grupos de " $x$ " personas se sientan en una mesa de capacidad " $y$ "

\begin{tabular}{|c|c|c|c|c|c|c|}
\hline $\begin{array}{c}\text { Tipo de } \\
\text { mesa }\end{array}$ & $\begin{array}{c}1 \text { persona } \\
\%\end{array}$ & $\begin{array}{c}2 \text { personas } \\
\%\end{array}$ & $\begin{array}{c}3 \text { personas } \\
\%\end{array}$ & $\begin{array}{c}4 \text { personas } \\
\%\end{array}$ & $\begin{array}{c}5 \text { personas } \\
\%\end{array}$ & $\begin{array}{c}6 \text { personas } \\
\%\end{array}$ \\
\hline Barra & 22 & 0 & 0 & 0 & 0 & 0 \\
\hline 2 & 19 & 19 & 0 & 0 & 0 & 0 \\
\hline 4 & 38 & 52 & 80 & 85 & 0 & 0 \\
\hline 6 & 21 & 29 & 20 & 15 & 100 & 100 \\
\hline
\end{tabular}

Elaboración propia.

No se consideraron a los grupos integrados por más de seis personas, ya que representan menos del $1 \%$ de la población de grupos de clientes. Anteriormente se mencionó la importancia que tiene la gestión de mesas debido a que en muchos casos gestionar al cliente para disminuir la duración del ciclo puede llevar a la desconformidad de este, por lo que la forma en la cual se determine la mejor mezcla de mesas para el restaurante repercutirá en el incremento de la utilización de los asientos y por ende en los ingresos del restaurante.

Después de analizar la complejidad del servicio del restaurante y de calcular las distribuciones en todas las etapas de atención al cliente, se procedió a construir el modelo de simulación utilizando el software ARENA, tomando como regla de decisión los siguientes supuestos:

a. Los grupos no son combinables; es decir, no se puede sentar a dos grupos de 2 personas en una mesa para cuatro.

b. Las mesas no son combinables; conocemos que las mesas del restaurante pueden ser combinadas para crear mesas cuya capacidad sea superior a 6 personas, sin embargo estamos haciendo uso de mesas rígidas y no combinables en el modelo con la finalidad de simular diferentes escenarios donde la distribución de las mesas sea diferente. 
c. Los tiempos de atención de los mozos, preparación de los ítems, consumo, pago y retiro de los clientes han sido calculados de acuerdo con la información obtenida del POS del restaurante así como los apuntes correspondientes a la toma de tiempos realizada en el restaurante.

La simulación se realizó por un período de 100 días con 20 réplicas, las horas en las cuales la simulación aceptará entrada de clientes van desde las 12:00 horas hasta las 17:00 horas, tomando en cuenta a los clientes que permanecen dentro del restaurante aún este haya cerrado sus puertas. Durante el período de captura de datos se observó que pese a que el restaurante atiende bajo la modalidad del restaurante 7 horas al día de lunes a sábado, se presentaron los casos de clientes que se quedaron charlando durante una hora extra por lo que el modelo de simulación considera que los clientes pueden quedarse dentro del restaurante hasta las 18:00 horas. Con esta observación se calculó la utilización de las mesas, ya que se tiene el total de horas en las cuales las mesas pueden ser utilizadas durante la simulación: 7 horas diarias x 100 días. Para realizar los cálculos de la utilización y de los ingresos se consideró lo siguiente:

a. El cálculo de la utilización se obtuvo sumando todos los minutos en los cuales las mesas fueron ocupadas por los clientes, y luego dividirlo entre las 7 horas en las que el restaurante atiende a sus clientes.

b. Para calcular los ingresos del restaurante se utilizó el consumo promedio, y de acuerdo al PAX que lo conforma se fueron sumando los valores.

\subsubsection{Escenarios de evaluación y mezcla de mesas}

Se consideraron 3 escenarios con diferentes reglas de gestión del cliente, la descripción de los cuales se detalla a continuación:

Escenario 1: Realidad del restaurante sin gestión de clientes, es decir, no se aplican reglas de decisión en la gestión del cliente.

Escenario 2: Regla de la "mesa superior" en la gestión de clientes; en este escenario un anfitrión ubica a los clientes en las mesas de acuerdo con el tamaño de su PAX, bajo la siguiente condición; en caso de que no existan mesas disponibles el anfitrión ubicará al cliente en la mesa con capacidad superior inmediata al tamaño del PAX. Es decir, si un grupo de dos personas llega al restaurante y el anfitrión lo intenta de ubicar en una mesa para dos personas, pero esta última no se encuentra disponible, el anfitrión lo ubicará en una mesa para cuatro personas, evitando que el cliente haga cola o se retire del restaurante.

Escenario 3: Regla de la "mesa exacta" en la gestión de clientes. Se define a esta regla como aquella donde se ubicará al cliente únicamente en la mesa que corresponda a su PAX. En caso de que no haya mesas disponibles el cliente entra 
automáticamente a la cola de espera, en la cual los clientes pueden decidir si esperan o se retiran, con una probabilidad de 0.50 para cada opción. Esta regla de gestión del cliente es un poco drástica, sin embargo, en el mercado peruano existen restaurantes que la aplican colocando carteles de "Reservado" en las mesas aunque no existan reservas en dichas mesas.

Por cada escenario se construyeron 3 mezclas de mesas diferentes para realizar la simulación. Las mezclas de mesas utilizadas son las siguientes:

Mezcla 1: Es la mezcla de mesas actual del restaurante, es decir, quince asientos en la barra para una persona cada uno, una mesa para dos personas, seis mesas para cuatro personas, y dos mesas para seis personas.

Mezcla 2: Quince asientos en la barra para una persona cada uno, doce mesas para dos personas, dos mesas para cuatro personas y una mesa para seis personas, debido a que la mayoría de grupos que ingresan al restaurante está conformado por dos personas.

Mezcla 3: Quince asientos en la barra para una persona cada uno, dos mesas para dos personas, cuatro mesas para cuatro personas y tres mesas para seis personas.

Siempre se consideró la barra con 15 asientos para cada escenario, ya que realizar cambios en la barra requeriría una inversión que los dueños del restaurante no están dispuestos a realizar por lo que aumentar o disminuir la capacidad en la barra no era una opción.

\subsubsection{Restricciones del modelo}

Para la simulación del modelo se establecieron las siguientes restricciones:

a. El modelo no contempla a los mozos como recurso, es decir, los mozos son ilimitados, por lo que no se generarán cuellos de botella por espera de atención o falta de mozos. No se simuló esta falta en la capacidad ya que durante la captura de datos no se presentó ningún caso en donde los mozos generaran cuellos de botella.

b. Los clientes no realizan pedidos por segunda o tercera vez ya que la información obtenida para las distribuciones se encuentra ya segmentada: preproceso, en proceso, posproceso.

c. No se consideraron los tiempos de espera en la cola, por lo que el cliente puede estar esperando en la cola desde unos cuantos minutos hasta varias horas debido a que el propósito de la simulación es conocer el impacto que tiene la mezcla de mesas y la gestión del cliente sobre los ingresos, y no sobre la gestión de las colas. 


\subsection{Resultados}

\section{Escenario 1. Realidad del restaurante sin gestión de clientes}

En este primer escenario, en el cual no existen reglas de decisión en la gestión del cliente, la mezcla de mesas con mayores ingresos corresponde a la mezcla 3, tal como se aprecia en la figura 2. La mezcla de mesas actual corresponde a la mezcla 1, por lo que si el restaurante decide no adoptar ninguna política de gestión de clientes, y bajo las condiciones mencionadas anteriormente, la mezcla de mesas más óptima sería de quince mesas con capacidad para una persona por cada mesa (barra), dos mesas para dos personas, cuatro mesas para cuatro personas y tres mesas para seis personas. El cambiar dos mesas para cuatro personas por una mesa para seis personas y una mesa para dos personas incrementa los ingresos, al reducir la cantidad de clientes perdidos y aumentando la cantidad de clientes atendidos.

Figura 2. Resultados de Escenario 1, software ARENA

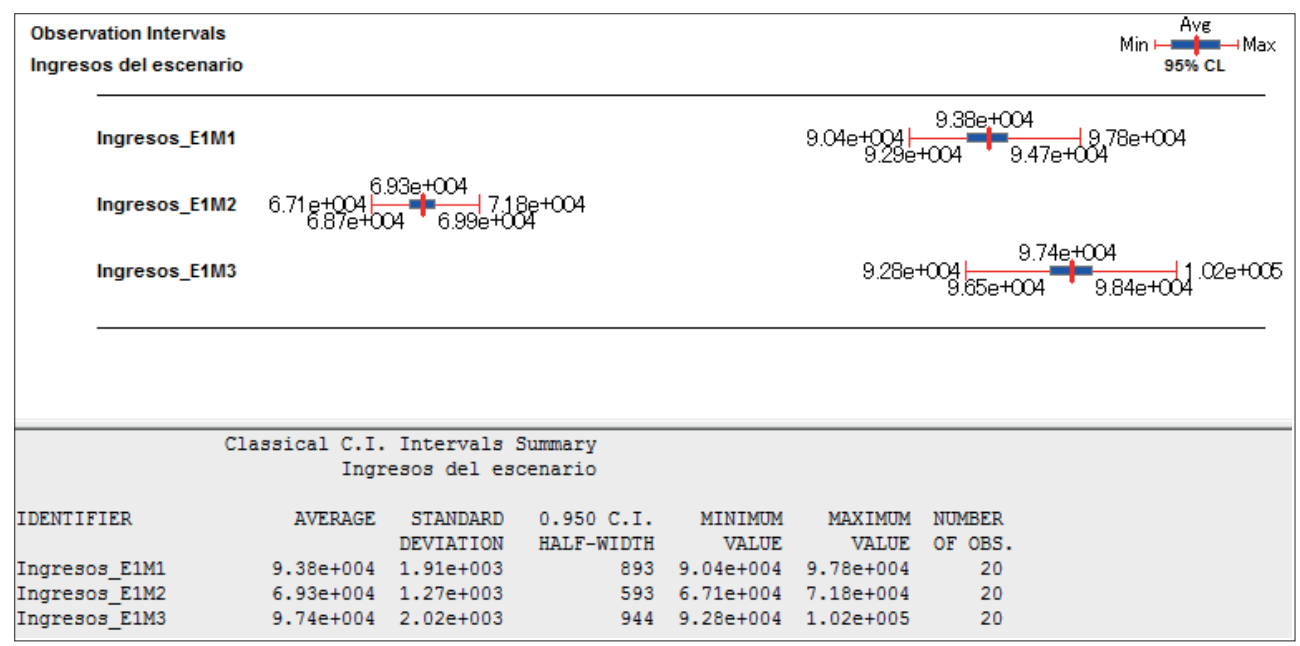

Elaboración propia. Captura de pantalla del software ARENA.

\section{Escenario 2. Regla de la "mesa superior" en la gestión de clientes}

En el siguiente escenario el modelo simula una política de gestión de clientes, donde se ubica a los clientes en mesas con una capacidad igual o superior al tamaño de su PAX. Bajo esta condición se simularon las tres mezclas de mesas descritas previamente, obteniéndose los siguientes resultados (figura 3): 
Figura 3. Resultados de Escenario 2, software ARENA

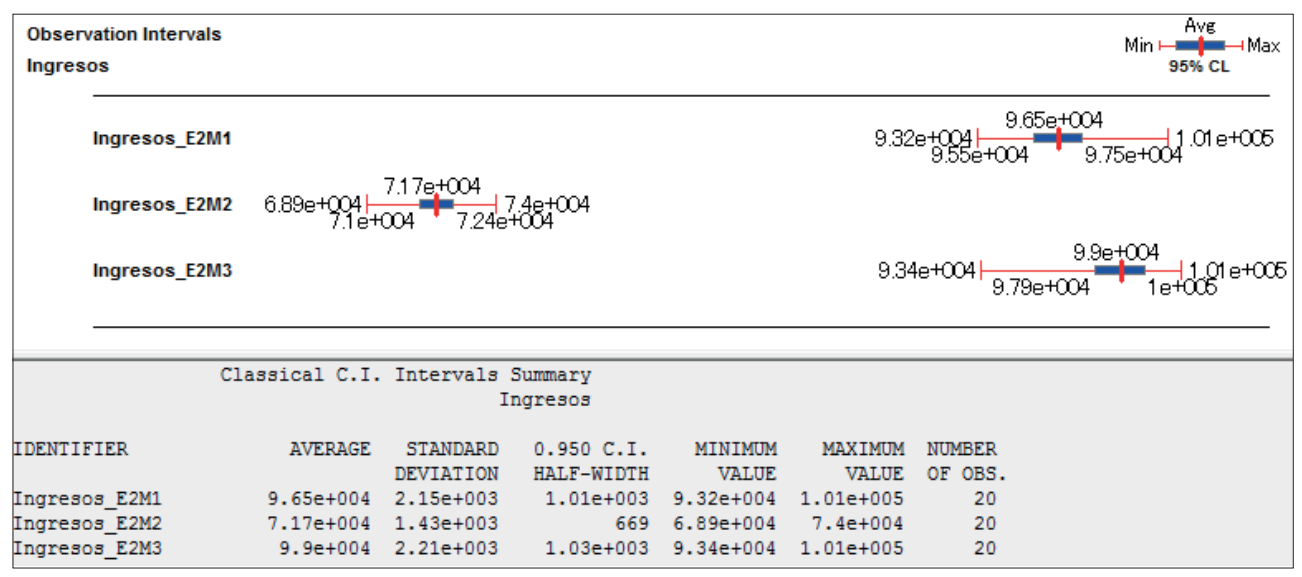

Elaboración propia. Captura de pantalla del software ARENA.

En este escenario se aprecia el impacto que tiene la gestión del cliente y la mezcla de mesas sobre los ingresos. Se observa que al aplicar la política de la "mesa superior" en la gestión del cliente, la mezcla 3 es aquella que tiene la menor cantidad de clientes perdidos, y los mayores ingresos. Por otro lado, el riesgo de aplicar de manera rigurosa cualquier política de gestión del cliente recae sobre un impacto negativo en la satisfacción del cliente. En otras palabras, sin importar el hecho de que el restaurante se encuentre aplicando una política de gestión de clientes, si un grupo de dos personas no desea ser acomodado en una mesa para dos, y desea sentarse en una mesa para cuatro o seis, entonces el anfitrión intentará persuadir al cliente, por lo cual la probabilidad de que se retire del restaurante aumenta debido a la disconformidad que representa. Por ello es importante conocer no solo la capacidad de las mesas con mayor demanda sino también su ubicación en el establecimiento para poder mejorar los ingresos sin impactar la satisfacción del cliente.

\section{Escenario 3. Regla de la "mesa exacta" en la gestión de clientes}

Bajo esta regla se simularon las 3 mezclas de mesas, obteniéndose los resultados que se aprecian en la figura 4: 
Figura 4. Resultados de Escenario 3, software ARENA

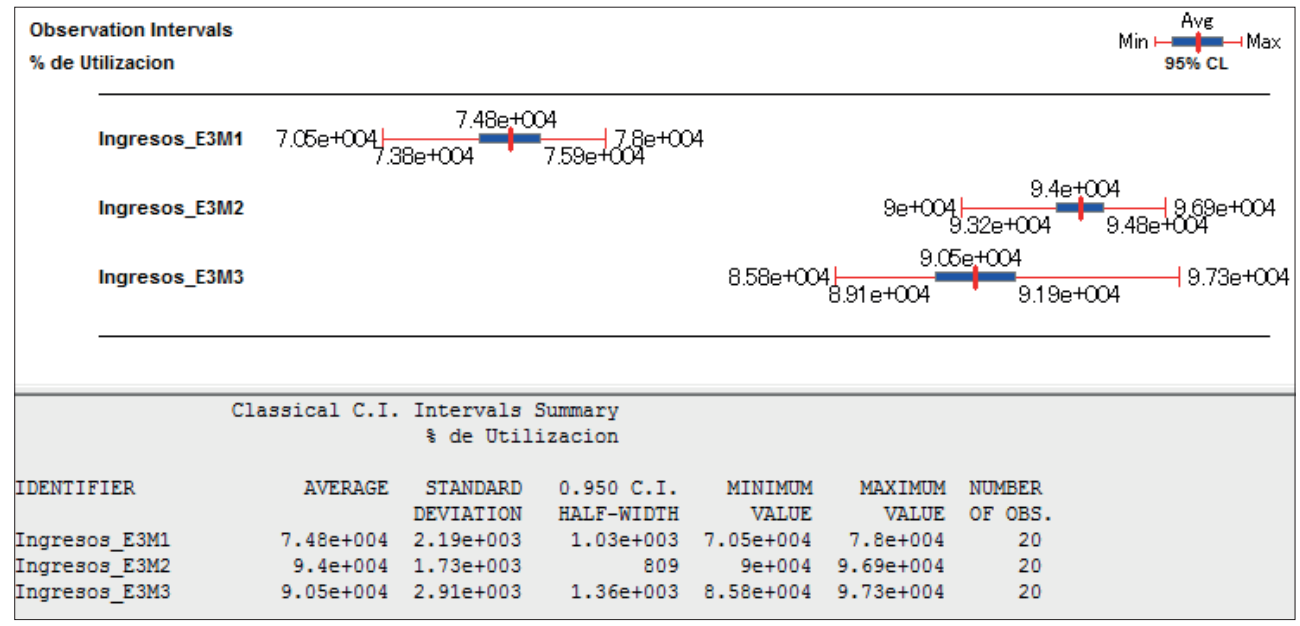

Elaboración propia. Captura de pantalla del software ARENA.

En este escenario se observa que al aplicar esta rígida política de gestión de clientes, la mezcla 2 de mesas es la más adecuada, ya que al realizar la comparación de los clientes atendidos con las mezclas 1 y 3, la mezcla 2 es la que tiene mayor número de atenciones. Por otro lado, los ingresos de la mezcla de mesas 2 se encuentran dentro del rango de la mezcla de mesas 3; sin embargo, la media de esta mezcla de mesas es superior a la de la mezcla de mesas 3.

\subsection{Conclusiones generales de la simulación}

Al cruzar la información de los ingresos generados por cada una de las 3 mezclas en los 3 escenarios propuestos se obtuvieron los resultados representados en la figura 5:

En resumen, se tiene lo siguiente:

a. En el escenario 1, sin gestión del cliente, la mezcla de mesas que genera mayores ingresos es la mezcla 3.

b. En el escenario 2, aplicando la política de gestión de la "mesa superior", la mezcla de mesas que genera mayores ingresos es la mezcla 3.

c. En el escenario 3, aplicando la política de gestión de la "mesa exacta", la mezcla de mesas que genera mayores ingresos es la mezcla 2. 
Figura 5. Resultados de todos los escenarios, software ARENA

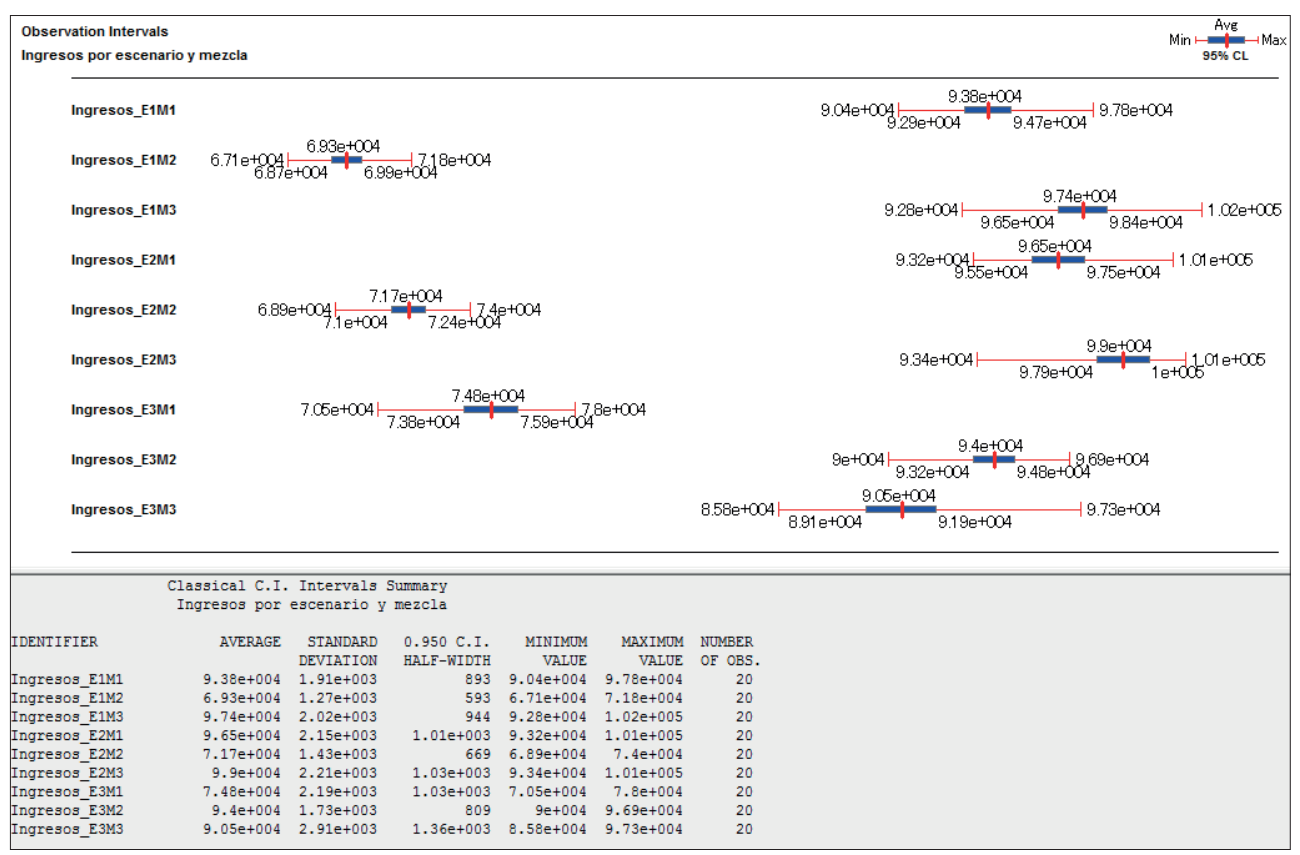

Elaboración propia. Captura de pantalla del software ARENA.

Se concluye que la implementación de cualquier política de gestión de clientes debe ser cuidadosamente observada para determinar la composición de la mezcla de mesas. Mediante este prototipo de soporte de decisiones se observa que aunque el mayor porcentaje de composición del PAX se encuentra relacionado con los grupos de dos personas, esto no implica que al aumentar las mesas con capacidad para dos personas vayan a aumentar los ingresos. Se observa que la mezcla 2, donde hay un mayor número de mesas para dos personas, no es la mezcla que presenta los mayores ingresos en los escenarios planteados, con excepción del escenario 3, cuya política de gestión de clientes es demasiado rígida.

Con los resultados obtenidos mediante la simulación realizada se propuso realizar los cambios pertinentes en la mezcla de mesas en el restaurante, de acuerdo con lo expresado en la mezcla 3, es decir, quince asientos en la barra para una persona cada uno, dos mesas para dos personas, cuatro mesas para cuatro personas y tres mesas para seis personas. Así como la implementación de la regla de la "mesa superior" en la gestión de clientes. Luego de dos meses de realizada la implementación se volvió a alcanzar los valores del indicador REVPASH, obteniéndose los resultados que se presentan en la tabla 13: 
Tabla 13. REVPASH obtenidos con la implementación de la mezcla 3 de las mesas

\begin{tabular}{|c|c|c|c|c|c|c|}
\hline Hora & Lunes & Martes & Miércoles & Jueves & Viernes & Sábado \\
\hline 12:00 - 13:00 & $\mathrm{S} / .10 .56$ & $\mathrm{~S} / 7.35$ & $\mathrm{~S} / .7 .80$ & $\mathrm{~S} / .6 .93$ & $\mathrm{~S} / .4 .25$ & $\mathrm{~S} / .2 .10$ \\
\hline $13: 00-14: 00$ & $\mathrm{~S} / .91 .66$ & $\mathrm{~S} / .69 .33$ & $\mathrm{~S} / .65 .36$ & $\mathrm{~S} / .93 .12$ & $\mathrm{~S} / .95 .55$ & $\mathrm{~S} / .35 .20$ \\
\hline $14: 00-15: 00$ & $\mathrm{~S} / .90 .60$ & $\mathrm{~S} / .78 .32$ & $\mathrm{~S} / 86.30$ & $\mathrm{~S} / .97 .36$ & $\mathrm{~S} / .102 .23$ & $\mathrm{~S} / .60 .11$ \\
\hline $15: 00-16: 00$ & $\mathrm{~S} / .36 .99$ & $\mathrm{~S} / .25 .76$ & $\mathrm{~S} / 22.74$ & $\mathrm{~S} / .33 .97$ & $\mathrm{~S} / 36.97$ & $\mathrm{~S} / .59 .85$ \\
\hline $16: 00-17: 00$ & $\mathrm{~S} / .7 .06$ & $\mathrm{~S} / .9 .35$ & $\mathrm{~S} / .2 .15$ & $\mathrm{~S} / 3.47$ & $\mathrm{~S} / .9 .88$ & $\mathrm{~S} / .15 .23$ \\
\hline $17: 00-18: 00$ & $\mathrm{~S} / 1.60$ & $\mathrm{~S} / .6 .23$ & $\mathrm{~S} / .0 .00$ & $\mathrm{~S} / .2 .25$ & $\mathrm{~S} / .2 .31$ & $\mathrm{~S} / .6 .90$ \\
\hline $18: 00-19: 00$ & $\mathrm{~S} / .0 .00$ & $\mathrm{~S} / .0 .00$ & $\mathrm{~S} / .0 .00$ & $\mathrm{~S} / .0 .00$ & $\mathrm{~S} / .0 .00$ & $\mathrm{~S} / .0 .52$ \\
\hline
\end{tabular}

Elaboración propia.

Con el nuevo REVPASH calculado se observa la mejora en la rentabilidad que se obtuvo con la implementación de la política sugerida para la gestión de mesas, regla de la "mesa superior", y la modificación de la mezcla de mesas del restaurante.

\section{Conclusiones y recomendaciones}

Mediante esta aplicación se comprobó la utilidad del Revenue Management como modelo de gestión para ser implementado en herramientas de soporte de decisiones, y el valor que su uso genera al estructurar la toma de decisiones. Las conclusiones y las recomendaciones a las que se llegaron a través del presente estudio fueron las siguientes:

a) Mediante la mejora en la mezcla de mesas se pueden incrementar los ingresos, el REVPASH y la utilización de los recursos, en este caso las mesas, en un restaurante. Esto da como resultado un modelo de gestión efectivo en el cual los restaurantes pueden trabajar. Cabe señalar que la mezcla de mesas tiene un rol muy importante en los ingresos de los restaurantes siempre y cuando existan políticas de gestión del cliente.

b) La industria de los restaurantes en el Perú se encuentra en una gran expansión debido al arte de la culinaria. Sin embargo, en esta investigación se comprobó que la aplicación de estos modelos de gestión tiene un impacto directo sobre la rentabilidad y posiblemente sobre su supervivencia, debido a la fuerte competencia que se ha generado en los últimos años, por lo que no solo es necesario poseer el arte de la culinaria, sino también realizar una gestión adecuada frente a las operaciones en estos negocios. 


\section{Referencias}

Hwang, J., \& Yoon, S.-Y. (2009). Where would you like to sit? Understanding customers' privacy-seeking tendencies and seating behaviors to create effective restaurant environments. Journal of Foodservice Business Research, 3(12), 219-233.

Kimes, S. E., Wirtz, J., \& Noone, B. M. (2002). How long should dinner take? Measuring expected meal duration for restaurant revenue management. Journal of Revenue and Pricing Management, 3(1), 220-233.

Kimes, S. E. (2004). Restaurant revenue management. Cornell Hospitality Reports, 2(4), 34.

Kimes, S. E., \&Thompson, G. M. (2004). Restaurant revenue management at Chevys: Determining the best table mix. Decision Sciences, 3(35), 371-392.

Padilla, J., \& Cossa, A. (2011). Sistemas de optimización de precios y rentabilidad (OPR) en restaurantes. Interfases, 4, 39-66.

Talluri, K. T., \& van Ryzin, G. J. (2004). The theory and practice of Revenue Management. Boston, USA: Kluwer Academic Publishers.

\section{Glosario}

- POS. Dispositivos y tecnologías que ayudan en la tarea de gestión de operaciones de un establecimiento comercial o de venta al público.

- Demand-Management Decisions (DMD). Hace referencia a las decisiones de ventas (dónde y cuándo vender, a quién y a qué precio) o a decisiones en las cuales se estima la demanda y sus características utilizando el precio y la capacidad de control de la demanda.

- Revenue Per Available Seat Hour (REVPASH). Indicador del Revenue Management en restaurantes. Como su nombre lo indica, es la utilidad que genera cada asiento disponible en un determinado momento del día. A diferencia de otros indicadores, como el ticket promedio, el REVPASH no solo cumple la función en términos monetarios sino también en términos de capacidad.

- PAX. Es el número de integrantes que tiene un grupo de clientes en los restaurantes o el número de personas que se encuentra ocupando alguna mesa del restaurante. Por ejemplo: la mesa 1 tiene un PAX de 4 personas. 